\title{
Atrial ERK $1 / 2$ activation in the embryo leads to incomplete Septal closure: a novel mouse model of atrial Septal defect
}

\author{
Che-Chung Yeh ${ }^{1+}$, Yanying Fan ${ }^{1+}$, Yi-Lin Yang ${ }^{1}$ and Michael J. Mann ${ }^{1,2^{*}}$
}

\begin{abstract}
Background: MEK1 mutation and activated MAPK signaling has been found in patients with RASopathies and abnormal cardiac development. Previous studies have suggested that regulation of fetal MAPK signaling is essential for normal cardiac development. We investigated the effect of active MEK1 overexpression on fetal atrial septal development.

Methods and results: An inducible double transgenic (DTg) mouse model was developed in which cardiacspecific fetal expression of a constitutively active form of human MEK1 (aMEK1) was induced primarily in the atrium via the withdrawal of doxycycline from the drinking water of pregnant mice. Atrial septal defect (ASD) was found in $51 \%(23 / 45)$ of DTg mice. Fifty-two percent (12/23) of ASD mice died before weaning, and surviving ASD mice exhibited hypertrophic hearts with enlarged right atria and decreased fractional shorting (40 $\pm 2 \%$ vs. $48 \pm 0 \%, p<0$. 05). The model mimicked human ASD in several key clinical features: severe ASD was associated with growth impairment; ASD-specific mortality was highest within the early postnatal period; despite an even distribution of ASD among the sexes, early mortality was significantly higher in males. The expression of aMEK1 and increased phosphorylation of ERK1/2 was documented via Western blot in DTg fetal hearts, with the largest increases seen in atrial tissue. In an alternative transgenic aMEK1 model with elevated atrial MKP3 expression and corresponding suppression of increases in ERK1/2 phosphorylation, animals did not develop ASD.
\end{abstract}

Conclusion: This new model of ASD suggests that enhanced atrial MEK1-ERK1/2 signaling during fetal development disrupts normal atrial septation, possibly regulated by the balance of ERK1/2 phosphorylation.

Keywords: Atrial septal defect, RASopathies, Mitogen-activated protein kinase, MAP kinase phosphatase, Mitogenactivated protein kinase pathway

\section{Background}

Atrial septal defects (ASD) are among the most common types of congenital heart disease (CHD), with an estimated incidence of 56-100 per 100,000 live births [1]. While mutations in GATA4, MYH6, NKX2-5 and TBX5 genes have been linked to the abnormal septation of atrial chambers, most patients with ASD are diagnosed without known etiologic causes [2-5]. However, the risk of a secundum defect is increased in siblings of ASD

\footnotetext{
* Correspondence: Michael.Mann@ucsf.edu

${ }^{\dagger}$ Equal contributors

${ }^{1}$ Cardiothoracic Translational Research Laboratory, University of California, San Francisco, San Francisco, CA, USA

${ }^{2}$ Division of Cardiothoracic Surgery, 500 Parnassus Avenue, Suite W420, San Francisco, CA 94143, USA
}

patients, suggesting that an inherited molecular mechanism may play a role in abnormal atrial septation.

The function of MEK1 signaling in the adult heart has been associated with "physiologic" hypertrophy [6]. Overexpression of active MEK1 induces hypertrophic changes in adult left ventricular (LV) structure that increase cardiac function and that do not degenerate into cardiomyopathy [6]. Increased MEK1 activity also protects hearts under stress conditions such as ischemiareperfusion and myocardial infarction $[7,8]$. In contrast to this positive influence of MEK1 activity in adult hearts, recent advances in the study of RASopathies have demonstrated a potentially detrimental role for Ras-RafMEK-ERK signaling during embryonic/fetal heart development [9]. RASopathies occur as a cluster of 
syndromes with germline mutations in genes participating in the Ras-Raf-MEK-ERK kinase signaling pathway. Independent of mutations and other syndromes, CHD including ASD - are common in RASopathies patients and are a major source of morbidity and even mortality [9]. Although mutations in human MEK1 genes have been identified in RASopathies, and the early application of a MEK1 inhibitor in animal models has been able to ameliorate associated defects [10-13], there is no direct evidence indicating how MEK1 may contribute to CHD.

We observed a very high incidence of ASD in double transgenic (DTg) mice in which expression of a constitutively active isoform of human MEK1 (aMEK1) regulated by an $\alpha$-myosin heavy chain $(\alpha M H C)$ promoter was induced during fetal development. Although the $\alpha \mathrm{MHC}$ promoter is generally used as a means to induce myocardial-specific gene expression in adult hearts, this promoter is also active in the embryonic/fetal heart with higher activity in the atrial region [14]. No previous model of relatively atrial-specific overexpression of aMEK1 in the developing fetus has been reported, nor are there reproducible, specific model.s of congenital ASD that closely mimic the human clinical scenario.

\section{Methods}

\section{Animals}

Hemagglutinin (HA)-tagged, constitutively active human MEK1 (aMEK1) cDNA, kindly provided by Dr. Natalie Ahn, was subcloned into the pTet-Splice vector (Invitrogen Corp.) for most of the experimentation reported, and also into the pTREtight vector (Clontech, Mountain View, CA) for validation of the transgenic model. The aMEK1 expression DNA cassettes were excised from both the pTet-Splice vector and the pTREtight vector, and each was used for pronuclear injection to generate founders carrying tetracycline responsive element regulated expression of aMEK1 gene (TAMEK). Genotyping was done by PCR using Primers 5' -GAGCTGGGGGCTGGCAATGG$3^{\prime}$ and $5^{\prime}$-CCTTGGCCTGGGTGGGGTCT-3'. Positive founders derived from each of the vectors were bred with C57BL/6 mice to obtained stable TAMEK transgenic ( $\mathrm{Tg})$ lines. The cardiac specific expression of tetracyclineresponsive transcriptional activator (tTA) $\alpha \mathrm{MHC}$-tTA $\mathrm{Tg}$ mice $(\mathrm{MH})$ were obtained from the Jackson Laboratory and were crossed with TAMEK mice to generate TAMEK/ MH DTg mice. The tTA transgene was detected by PCR using Primers 5 '-CGCTGTGGgGCATTTTACTTTAG$3^{\prime}$ and 5'-CATGTCCAGATCGAAATCGTC-3'. DTg mice and breeding pairs were treated with Doxycycline (DOX, $0.5 \mathrm{mg} / \mathrm{ml}$ ) in drinking water for suppression of transgene expression. DOX was withdrawn from the drinking water to induce fetal aMEK1 transgene expression. Wild type and /or single Tg littermates were used as controls for DTg mice. The MEK1 transgenic mouse, in which aMEK1 is driven by the $\alpha \mathrm{MHC}$ promoter without DOX regulation, was kindly provided by Dr. Jeffrey Molkentin [6]. Time mating was validated by checking the vaginal plugs in the morning and marking as 0.5 day post copulation $(\mathrm{dpc})$. All animals were maintained at a $12 \mathrm{~h}$ light-dark cycle with ad libitum access to food and water. All procedures conformed to the Guide for the Care and Use of Laboratory Animals published by the US National Institutes of Health (NIH Publication No. 85-23, revised 1996), and were approved by the Institutional Animal Care and Use Committee of the University of California, San Francisco. Please see Additional file 1 S1 for additional details on Methods.

\section{Anatomy and histology analysis}

Mouse hearts were arrested in diastole with i.p. injection of $1 \mathrm{ml}$ of $1 \mathrm{M} \mathrm{KCl}$ in saline, formalin-fixed and embedded in paraffin. To assess the morphology of the atrial septum, the right atrial appendage was removed, and the anatomical structure of septal wall was assessed under dissection microscopy. To document ASD and possible ventricular septal defects, serial continuous sections of paraffin embedded hearts were stained with Gomori's trichrome reagent [8]. Stained sections were reviewed for occurrence of septal defect and photographic images were acquired.

\section{Western blotting assay}

Fetal hearts were collected from fetuses at 10.5 to 14.5 $\mathrm{dpc}$; some fetal hearts were then divided into atrial and ventricular portions. Each tissue sample was homogenized in lysis buffer containing $150 \mathrm{mM} \mathrm{NaCl}, 50 \mathrm{mM}$ Tris- $\mathrm{HCl}, 1 \mathrm{mM} \mathrm{Na} 3 \mathrm{VO} 4,5 \mathrm{mM} \mathrm{NaF}, 1 \% \mathrm{NP} 40$, and protease inhibitor cocktail tablet (Roche Diagnostics, Indianapolis, IN). Samples containing equal amounts of protein were separated by NuPAGE Novex Bis-Tris Gels (Invitrogen, Carlsbad, CA) and transferred to PVDF membranes (Invitrogen). Blots were first probed with antibodies against phospho-ERK1/2 (P-ERK1/2, rabbit monoclonal, 1:2000, \#4376, Cell Signaling, Danvers, MA,) or total ERK1/2(rabbit polyclonal, 1:4000, \#9102, Cell Signaling), total MEK1/2 (rabbit monoclonal, 1:4000, \#9126, Cell Signaling), MKP1(rabbit polyclonal, 1:1000, SC-1102, Santa Cruz Biotech, Santa Cruz, CA), MKP3(mouse monoclonal, 1:1000, SC-1000374, Santa Cruz Biotech), tTA (mouse monoclonal anti-TetR, 1:2000, \#631131, Clontech, Mountain View, CA), GAPDH (rabbit polyclonal, sc-25,778, Santa Crus Biotech) or HA (mouse monoclonal, 1:2000, \#2367, Cell Signaling), and then with appropriate horseradish peroxidase-conjugated secondary antibodies (goat antirabbit IgG-HRP, 074-1056 and goat anti-mouse IgGHRP, 074-1806, KPL, Gaithersburg, MD). SuperSignal West Femto Maximum Sensitivity substrate (Thermo Fisher Scientific Inc., Waltham, MA) and Immobilon 
Western HRP Substrate (EMD Millipore, Billerica, MA) were used for the chemiluminescent visualization of proteins. Exposed films were then subjected to density analysis using Image J software (NIH).

\section{Echocardiography}

Transthoracic echocardiography was performed in conscious 8-week old female mice using an Acuson Sequoia 512 machine and a $13-\mathrm{MHz}$ probe [8]. A two dimensional short-axis view of the left ventricle was obtained at the level of the papillary muscles. Two-dimensional M-mode tracings were also recorded. LV fractional shortening (FS) and ejection fraction (EF) were calculated using the following equation: FS $(\%)=100 \times(\mathrm{LV}$ end of diastolic dimension - LV end of systolic dimension) /LV end of diastolic dimension; EF $(\%)=100 \times($ end of diastolic volume - end of systolic volume)/end of diastolic volume.

\section{Statistics}

Results are expressed as mean \pm SEM. Mean values were compared by the unpaired 2-tailed Student's $t$ test or ANOVA. Mortality rates were compared by
Fisher's exact test. $P$-values less than 0.05 were considered statistically significant. Although we examine many potential effects, we report nominal $p$-values, without adjustment for multiple testing. Such adjustment would be focused on avoidance of one or more results with $p<0.05$ in the case where all differences are truly zero [15-17], which is an extremely unrealistic hypothesis about the state of nature in our situation. We therefore rely on scientific judgment rather than formal adjustment methods to indicate where caution is warranted despite findings with $p<0.05$. In addition, adjustment would require that each result detract from the others, but there are clear biological relationships among the issues that we examine, and these permit coherent sets of findings to reinforce each other rather than detract from one another [18].

\section{Results}

Doxycyline induced aMEK1 expression and activity in TAMEK/MH double transgenic mice (DTg)

Even though $\alpha \mathrm{MHC}$ expression is traditionally associated with the adult heart, previous studies have

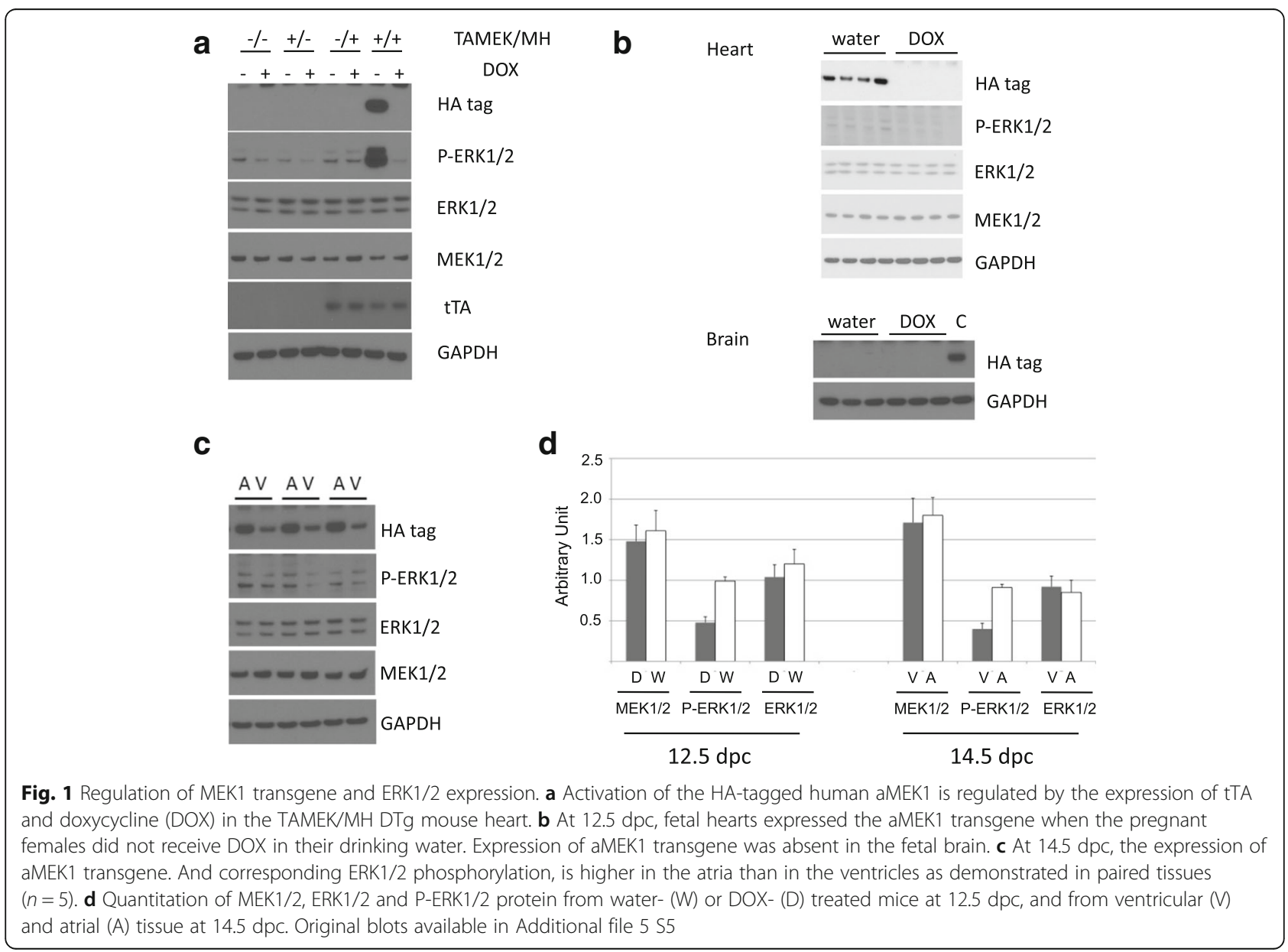


demonstrated the expression of this $\mathrm{MHC}$ isoform and the activity of its promoter in fetal hearts [14]. In the absence of DOX in the drinking water of pregnant mice, expression of the HA-tagged human aMEK1 transgene, dependent on tTA expression driven by the $\alpha \mathrm{MHC}$ promoter, was observed in DTg fetuses. Its expression was subsequently suppressed in DTg fetuses via introduction of DOX into the drinking water of pregnant mice (Fig. 1A). Although expression of the aMEK1 transgene was detectable via identification of an otherwise absent HA tag, the level of expression achievable in the fetal heart as driven by a combination of the relatively low activities of the $\alpha \mathrm{MHC}$ promoter and the tTA promoter in the fetal heart was not strong enough to yield a measurable increase in total MEK1 protein. As has been observed previously, however [19], the constitutive activity even of this relatively smaller amount of aMEK 1 protein compared to the total pool of non-phosphorylated and non-activated endogenous MEK1 resulted in a measurable increase in downstream ERK1/2 activation (i.e., phosphorylation, Fig. 1A-C).

During fetal development, aMEK1 transgene expression was identified in 14.5 dpc hearts, but aMEK1 expression was absent in the fetus' brain tissue (Fig. 1B). Furthermore, there was a differential expression pattern of aMEK1 transgene even within the developing fetal hearts. Expression of the HA-tagged aMEK1 gene, as well as increased phosphorylation of ERK1/2, was significantly higher in atrial tissue than in ventricular tissue from $14.5 \mathrm{dpc}$ hearts of DTg fetuses (Fig. 1C).

\section{Atrial septal defect in DTg in the absence of DOX (and in the presence of upregulated ERK $1 / 2$ phosphorylation)}

Hearts from a large number of DTg mice (23 out of 45 ) that had not been exposed to DOX in utero exhibited enlarged right atria compared to the right atria from their non-DTg littermates (Fig. 2A). Further anatomical analyses of 8 week-old hearts with hypertrophied right atria revealed the large, unfused foramen ovale connecting both atrial chambers, resembling secundum atrial septal defects (Fig. 2B, Additional file 2 S2). In contrast, only small vestigial foramena ovale were observed in WT hearts (Fig. 2B). The ASDs in DTg hearts were confirmed on whole heart histologic sections taken at 14 days of age, although no other ventricular or atrioventricular abnormalities were observed in $\mathrm{DTg}$ hearts, with or without in utero exposure to DOX (Fig. 2C).

\section{Premature mortality and compromised cardiac function in} DTg

In the absence of DOX exposure, crossbreeding of heterozygous TAMEK1 and $\mathrm{MH}$ mice yielded pups with four different transgenic genotypes in a classic Mendelian distribution (Fig. 2C). By postnatal day 21, however, only $73 \%(33 / 45)$ of the DTg pups had survived, while there was no mortality among the other three genotypes (Fig. 3A). Although the incidence of ASD was similar in male and female DTg in the early postnatal period, mortality was observed in 11/14 (79\%) of male ASD-DTg's at 21 days, compared to only $1 / 9$ (11\%) of female ASDDTg's at that time point ( $P=0.003$, Fig. 3B).

Even though DTg pups as a whole had similar initial birth bodyweights compared to their non-DTg littermates, postnatal growth of DTg pups subsequently diagnosed with ASD, but not DTg pups without ASD, was delayed at day $14(6.1 \pm 0.4 \mathrm{~g}$ for ASD-DTg vs. either 8.2 $\pm 0.2 \mathrm{~g}$ for non-DTg or $8.2 \pm 0.5 \mathrm{~g}$ non-ASD DTg, $p<$ 0.01 , Fig. $2 \mathrm{C} \& 3 \mathrm{C}$ ). Among the 14-day old DTg pups with ASD, the average bodyweight of mice that survived at wean (21 days) was marginally higher than those mice that did not subsequently survive $(7.1 \pm 0.4 \mathrm{~g}$ vs $6.1 \pm$ $0.4 \mathrm{~g}, P=0.07)$, suggesting a link between severity of the ASD and early postnatal growth. This early growth impairment may therefore serve as a diagnostic marker for severe ASD in this mouse model.

At 8 weeks of age, the body weight difference between surviving female ASD mice and other genotype/phenotype female mice had disappeared (we included only female groups in our statistical analysis due to the insufficient number of surviving male ASD-DTg mice, Table 1), reflecting better overall compensation for ASD in surviving ASD mice. All single transgenic and WT mice survived past the time of wean. Echocardiography was performed on 8-week old female mice from the four different genotypes. Compared to the non-ASD groups, the ASD-affected female DTg mice, despite equivalent body weights, had significantly decreased LV function as reflected both in lower fractional shortening $(40 \pm 2 \%$ vs $48 \pm 0 \%, p<0.05)$ and reduced ejection fraction $(67 \pm 2 \%$ vs. $75 \pm 0 \%, \mathrm{p}<0.05$; Table 1, Additional file $3 \mathrm{~S} 3$ ).

\section{Differential phosphorylation of ERK $1 / 2$ in the developing atria of two different aMEK1 transgenic models correlates to differential development of ASD}

In addition to the DTg model of inducible, cardiacspecific aMEK1 expression described here, our laboratory has also studied an alternative transgenic model, the MEK1 Tg mouse, in which cardiac-specific expression of aMEK1 is constitutive under the $\alpha M H C$ promoter and is not affected by DOX. In contrast to the inducible TAMEK/MH DTg model, the constitutively expressing MEK1 $\mathrm{Tg}$ mouse (a generous gift from the Jeffrey Molkentin laboratory) did not develop ASD or other congenital cardiac defects. We evaluated fetal transgene expression in the MEK1 Tg model, and did observe aMEK1 expression in $14.5 \mathrm{dpc}$ fetal hearts as 


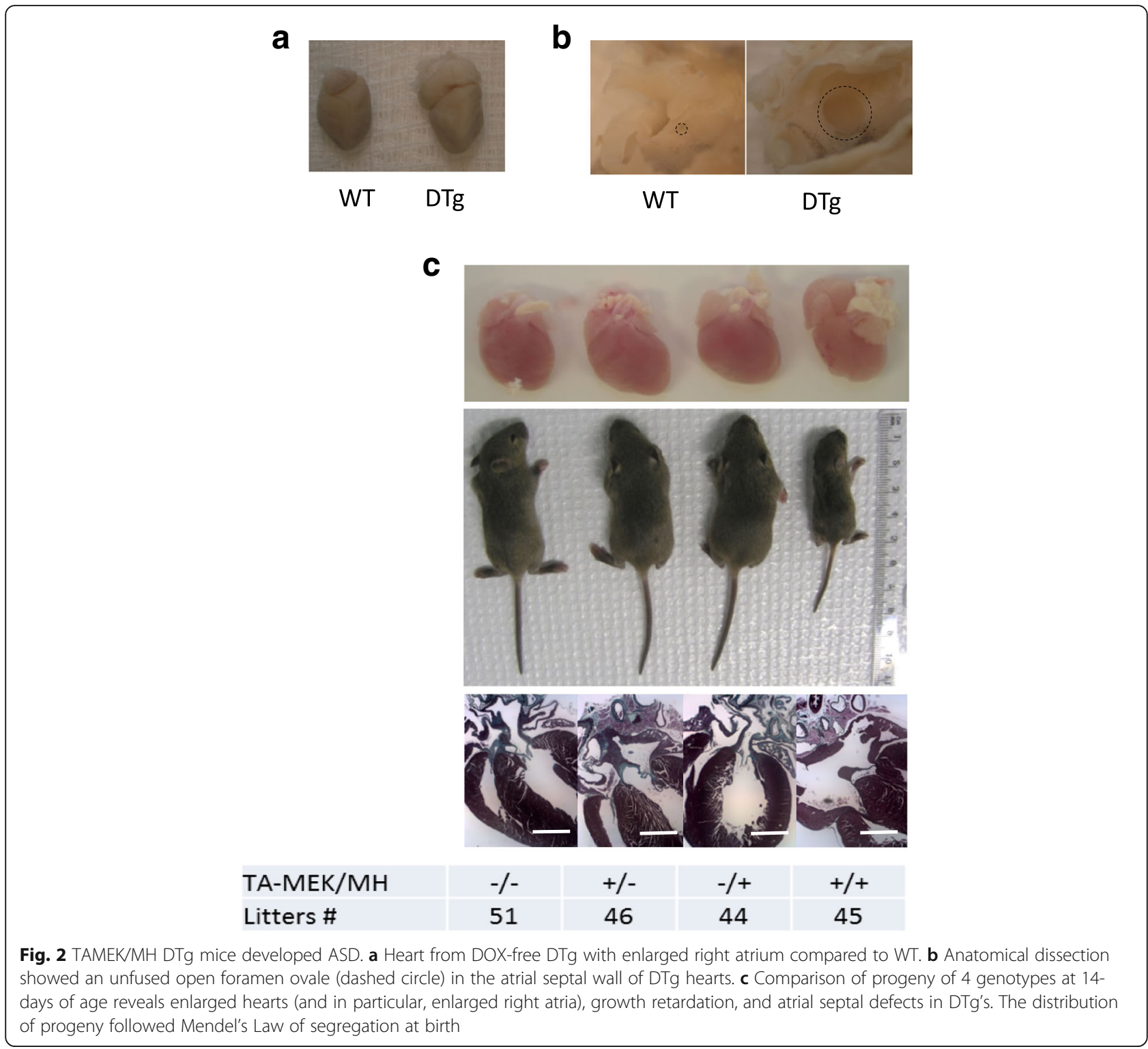

reflected by an increase in total MEK1 protein (Fig. 4). As in the TAMEK/MH DTg model, the $14.5 \mathrm{dpc}$ expression of aMEK1 in the constitutively expressing transgenic was also higher in the atria than in the ventricles. However, the pattern of atrial phosphorylation of ERK1/ 2 was markedly different in the two transgenic models. Although aMEK1 expression in adult MEK1 Tg hearts is associated with increased ERK1/2 expression and phosphorylation [6], neither atrial nor ventricular phosphorylation of ERK1/2 was significantly increased in heart tissues from constitutively expressing fetal $14.5 \mathrm{dpc}$ MEK1 Tg mice compared to their $14.5 \mathrm{dpc}$ WT siblings (Fig. 4). This difference in downstream ERK1/2 activation despite similar fetal atrial aMEK1 expression in the MEK1 Tg and our novel TAMEK/MH DTg mice correlated with the different phenotypes observed with regard to congenital
ASD development, as ASD was only observed in DTg mice in which atrial ERK1/2 phosphorylation was greater than that of both ventricular tissue from the same hearts and heart tissues from non-DTg littermates,.

In order to evaluate the possible roles of integration site and the use of different transgene expression vectors in the different molecular and clinical phenotypes observed in TAMEK/MH DTg and MEK1 Tg mice, we developed a second inducible model using the pTREtight vector. We observed a similar incidence and clinical impact of ASD in this second DTg line (Additional file 4, S4).

We also observed another interesting difference between the inducible TAMEK/MH DTg and the constitutively expressing MEK1 mouse with regard to MEK/ERK regulation. Expression of the negative regulator of ERK phosphorylation/activation, MAP kinase phosphotase 3 


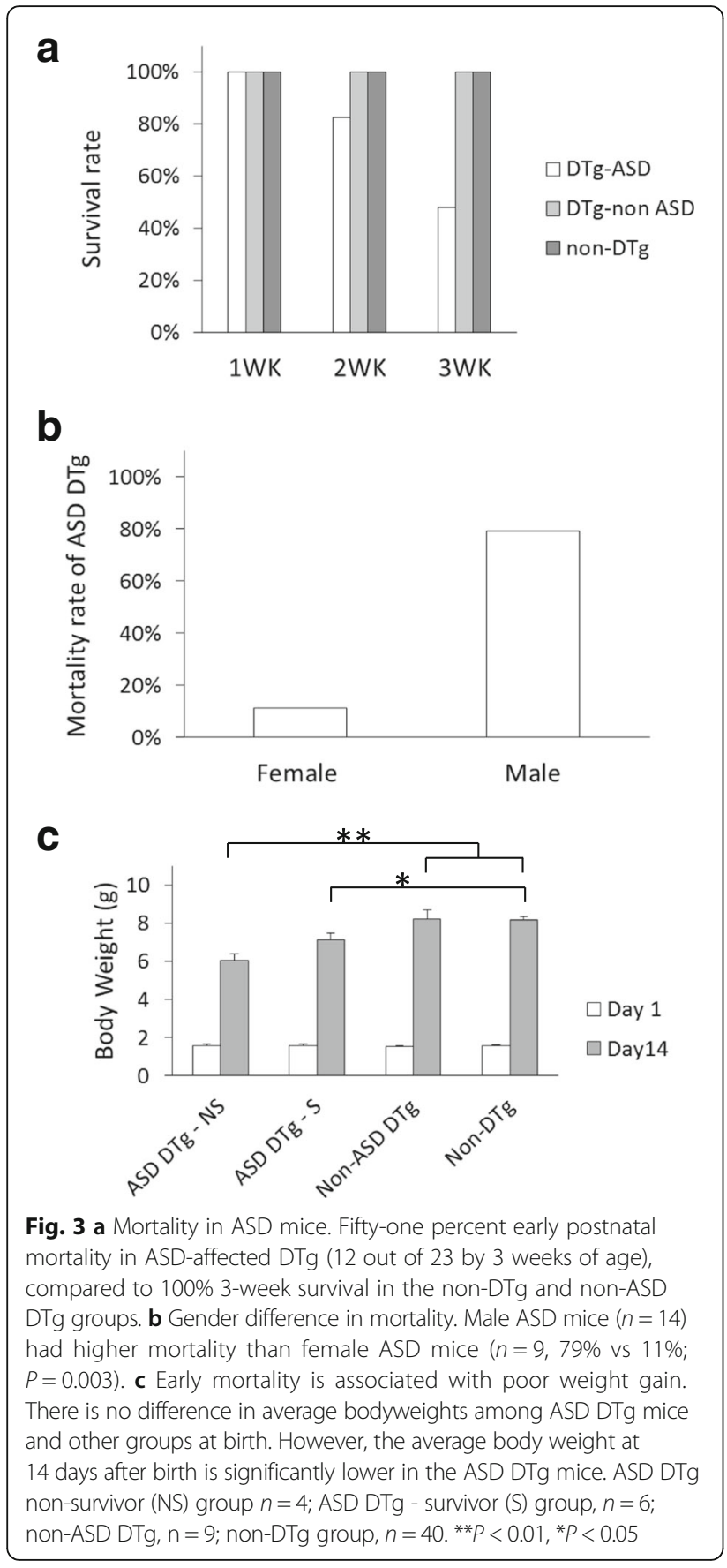

(MKP3) [19] was significantly higher in the atria and ventricles of $14.5 \mathrm{dpc}$ TAMEK/MH DTg mice than in cardiac tissues from their WT siblings (Fig. 4, Additional file 4 S4, $P<0.05)$. However, a similarly high level of MKP3 expression was observed in both (constitutively expressing) MEK1 Tg mice and in their respective WT siblings (Fig. 4). In contrast, the expression of MKP1, which also regulates p38 and JNK phosphorylation [20], was similar among atrial and ventricular tissues from all four groups of mice (Fig. 4).
Table 1 Echocardiographic measurements of LV function in 8week old mice bearing different transgenic genotypes

\begin{tabular}{lllll}
\hline $\begin{array}{l}\text { Genotype } \\
\text { (TAMEK/MH) }\end{array}$ & $-/-$ & $+/-$ & $-/+$ & $+/+$ \\
\hline BW(g) & $22 \pm 1$ & $21 \pm 0$ & $21 \pm 0$ & $22 \pm 0$ \\
EF(\%) & $75 \pm 0$ & $75 \pm 0$ & $76 \pm 0$ & $64 \pm 2^{*}$ \\
FS(\%) & $48 \pm 0$ & $48 \pm 0$ & $48 \pm 0$ & $37 \pm 2^{*}$ \\
IVSd (mm) & $0.51 \pm 0.05$ & $0.53 \pm 0.06$ & $0.51 \pm 0.05$ & $0.48 \pm 0.05$ \\
IVSs (mm) & $0.65 \pm 0.11$ & $0.66 \pm 0.11$ & $0.65 \pm 0.10$ & $0.60 \pm 0.09$ \\
LVAWd (mm) & $0.80 \pm 0.06$ & $0.83 \pm 0.06$ & $0.79 \pm 0.05$ & $0.78 \pm 0.06$ \\
LVAWs (mm) & $1.15 \pm 0.09$ & $1.15 \pm 0.08$ & $1.11 \pm 0.08$ & $1.10 \pm 0.08$ \\
LVIDd (mm) & $3.70 \pm 0.08$ & $3.74 \pm 0.09$ & $3.67 \pm 0.07$ & $4.11 \pm 0.09^{*}$ \\
LVIDs (mm) & $2.51 \pm 0.13$ & $2.55 \pm 0.10$ & $2.48 \pm 0.09$ & $2.51 \pm 0.10^{*}$ \\
$n$ & 5 & 5 & 5 & 5
\end{tabular}

$B W$ body weight, $E F$ ejection fraction, FS fractional shortening, IVS interventricular septum, LVAW left ventricular anterior wall, LVID left ventricular internal dimension; +/+ group includes ASD mice only; please see Additional file 6 S 6 for data from $+/+$ non-ASD mice. ${ }^{*} P<0.05$

\section{Discussion}

The severity of human ASD differs drastically among affected individuals, ranging from life-threatening to symptomless. Although ASD is one of the most common congenital heart defects, this clinical variability in phenotype makes identification of factors that interrupt normal atrial septal development even more difficult. There are several genetic mouse models that exhibit defects in atrial septation. Among the most often reported are models of functionally impaired or heterozygous null NKX2-5 and GATA4, based largely on early associations of mutations in these genes for these transcription factors with human ASD and other congenital defects [2, 3, 21-23]. These models of transcription factor disruption, however, have proven to be extremely complex, as their mechanisms involve disruption not only of the target transcription factor but also impaired expression of other related transcription factors such as TBX5 and MEF2c [21]. As a result, changes in numerous downstream effectors regulated by these multiple transcription regulators are at play. Confounding the mechanistic analyses of these models is their frequent association with extracardiac abnormalities, and the fact that they are often embryonic lethal and demonstrate compound septal defects involving both atria and ventricles.

In contrast, the model described here of isolated congenital ASD offers a relatively focused mechanistic pathway for future study; it is directed to a wellcharacterized signaling pathway rather than a complex of pleiotropic upstream transcription factors. In addition to the mechanistic complexity of previously reported genetic models of ASD, the perinatal characteristics of those models do not closely mimic the clinical findings 


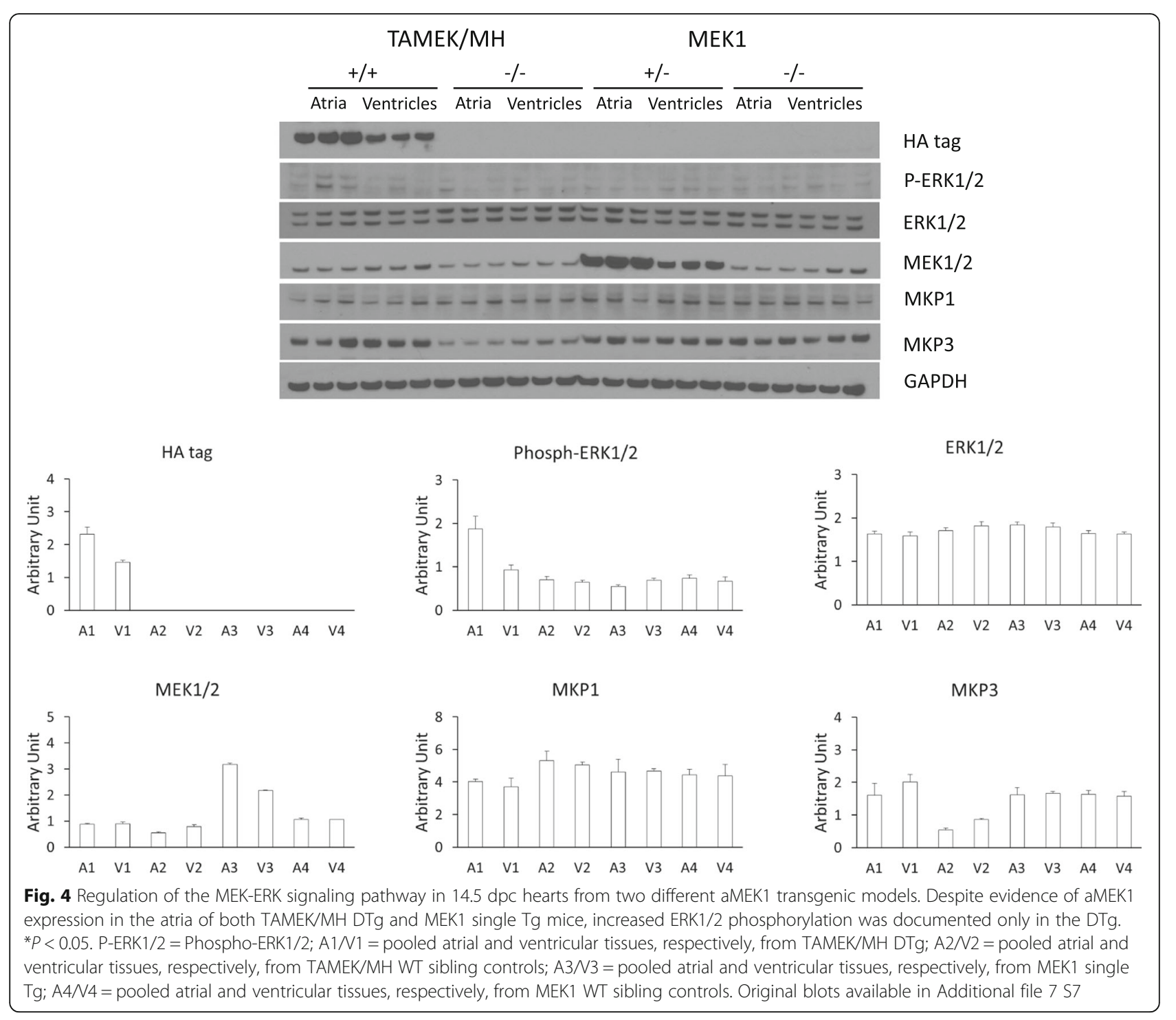

in human ASD patients. Our novel model of isolated ASD, however, resembles human ASD in the following important ways: severe ASD is associated with growth impairment; ASD-specific mortality is highest within the early postnatal period; although the incidence of ASD is evenly distributed among the sexes, this early mortality is significantly higher in males ([24, 25]; Table 2).

Our current mouse model likely reflects an important role of Ras-MEK-MAPK signaling in the development of RASopathy syndromes, including atrial septal defects. In fact, we have observed for the first time that upregulation of MEK-ERK signaling in an atrial-specific manner, resulting in higher atrial ERK1/2 phosphorylation, is associated with abnormal atrial septation. Furthermore, by comparing two aMEK1 transgenic mouse models, we substantiated the potential role for site-specific ERK1/2 activation in the development of ASD.

Since MAPK signaling pathways can be regulated by various stimuli, such as growth factors, hormones, exogenous chemicals and even mechanical stresses [26-30],

Table 2 Similarities between human ASD and the MEK1-mediated mouse ASD model

\begin{tabular}{lll}
\hline & Human ASD & MEK1 ASD mouse model \\
\hline Gender distribution at birth & $51 \%$ male & $56 \%$ male \\
Gender dependent long term survival ate & $65 \%$ survivors were female ( $>5$ years) & $89 \%$ survivors were female (21 days) \\
Major mortality stage & Early, first year after birth (75\% of total death) & Early, before weaning (100\% of death till 8wk old) \\
Growth impairment & Weight and length & Weight and length \\
\hline
\end{tabular}


our findings also suggest that genetic-independent, aberrant activation of ERK1/2 during pregnancy may lead to the sporadic occurrence of ASD.

The mechanism by which upregulated ERK1/2 activation is absent in the fetal atria of the constitutively expressing MEK1 mouse, despite fetal cardiac aMEK1 expression driven by the $\alpha \mathrm{MHC}$ promoter, remains unclear. One explanation for differences in phenotype between the two transgenic models studied may be related to differences in integration site and the use of different transgene expression vectors in their generation. However, we observed a similar incidence and clinical impact of ASD in a second DTg line derived via a distinct integration using an alternative vector.

It is also possible that the difference in genetic backgrounds between the double transgenic (C57Bl/6-FVBN mix in both lines studied) and single transgenic (pure FVBN) mouse models may modulate the downstream effects of aMEK1 transgene expression. The importance of genetic background as a possible regulator of congenital defects has been demonstrated previously in the Nkx2-5 transgenic mouse [31]. Heterozygous $N k x 2-5$ knockout mice in the inbred strain background C57Bl/6 frequently developed atrial and ventricular septal defects, whereas the incidences of these defects were substantially reduced in the $N k x 2-5(+/-)$ progeny outcrossed to the strains $\mathrm{FVB} / \mathrm{N}$ or A/J [31]. Since the pure FVBNderived single Tg MEK1 and its WT siblings both had higher fetal cardiac MKP3 expression than the WT siblings of the $\mathrm{C} 57 \mathrm{Bl} / 6-\mathrm{FVBN}$-derived $\mathrm{DTg}$ or control $\mathrm{C} 57 \mathrm{Bl} / 6$ mice, it is possible that the expression level of MKP3 is related to this difference in genetic backgrounds. Higher pre-existing MKP3 levels in the fetal atria of MEK1 single Tg hearts may have suppressed activation of ERK1/2, thereby resulting in resistance to ASD despite aMEK1 transgene expression (Fig. 5). The higher levels of MKP3 observed in the DOX-withdrawn DTg may, in turn, reflect a feedback loop that is not as effective as high constitutive MKP3 expression in controlling ERK1/2 activation. In fact, previous studies have indicated that MKP3-mediated suppression of ERK activation is required for proper development of the kidneys, neural plate and limb bud mesenchyme [32-34]. A similar mechanism may be at play to explain why lower levels of $\alpha$ MHC-driven aMEK1 expression in fetal MEK1 Tg hearts did not exhibit the increased ERK1/2 phosphorylation seen in adult MEK1 Tg hearts with significantly higher $\alpha M H C$-driven aMEK1 expression. Studies in MKP3-null mouse lines derived from mice with different genetic backgrounds have also demonstrated variable influences on development even with similar increases in ERK phosphorylation [35, 36]. The results reported here do suggest that the fine tuning of ERK1/2 signaling via MKP3 expression levels at specific locations within the developing heart may be essential for the proper organ and tissue development [37].

Developmental defects in the brain have also been observed in models of upstream ERK activation via Braf Q241R or Neurofibromatosis type 1 gene overexpression. Treatment with MEK inhibitors was found to correct developmental defects in these experimental animals [11-13, 38]. While ERKs are known to be preferentially activated both in the regions of the primitive brain and in the heart during early development, our new evidence underscores the importance of carefully regulated MAPK signaling during fetal development. Any dysregulation or over-activation of this pathway that leads to an increase in ERK1/2 phosphorylation may
MEK1 Tg

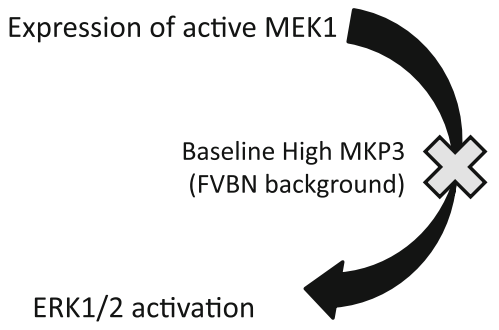

TAMEK/MH DTg

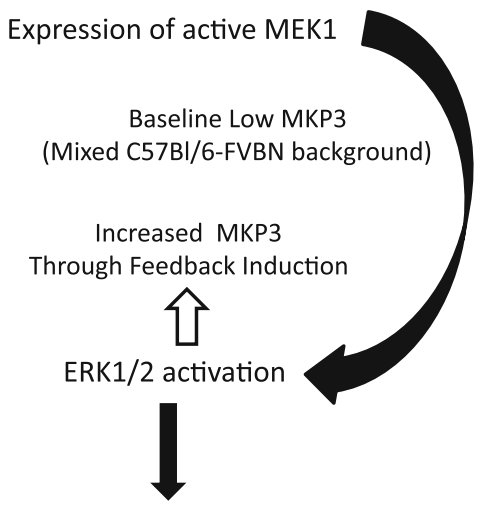

ASD

Fig. 5 Proposed role of baseline MKP3 expression in determining ERK1/2 activation and, in turn, putative induction of ASD phenotype in transgenic mice with expression of constitutively active MEK1 (see Discussion) 
impact the normal development of specific organ structure or function.

\section{Conclusions}

Our data indicate that the regulation of MAPK signaling is essential for normal development of the atrial septum. Specifically, atrial upregulation of activated ERK1/2 was associated with failure of normal septal closure. The TAMEK1/MH DTg mouse is the first ASD-specific model that closely mimics the clinical manifestations of human congenital ASD, and therefore may serve as a novel tool to elucidate both the development of human fetal septal defects as well as possible therapeutic interventions. Furthermore, our data also begin to suggest that modifiers of MAPK signaling in fetal hearts, such as MKP3 and other internal/environmental factors, should be further evaluated to better understand the complex, multifactorial development of ASD and other congenital defects.

\section{Additional files}

Additional file 1: S1 Methods. (DOCX $34 \mathrm{~kb})$

Additional file 2: S2 Figure. Comparison of progeny of 4 genotypes at 8 weeks of age showed atrial septal defect in DTg (+/+). (PPTX $1404 \mathrm{~kb})$

Additional file 3: S3 Figure. Survived ASD affected DTg (+/+, from line \#25) and its non- DTg siblings at 8 weeks old. (PPTX $206 \mathrm{~kb}$ )

Additional file 4: 54 Figure Anatomical dissection revealed ASD in two independent DTg lines at 8 weeks old. A. line \#25, derived from expression sequence using pTet-Splice vector; B. line \#8, derived from expression sequence using pTREtight vector. C. MEK1/2, P-ERK1/2, ERK1/2, MKP1, and MKP3 protein levels as detected by Western blot in atrial (A) and ventricular (V) tissue from $14.5 \mathrm{dpc}$ DTg (+/+) and WT (-/-) littermates from line \#8 (C57BI/6-FVBN mixed background) and MKP1, and MKP3 from control pure C57BI/6 mice. D. Survival among DTg mice from line \#8 with $(n=11)$ and without $(n=12)$ ASD, and among non-DTg littermates $(n=66)$. (PDF $1225 \mathrm{~kb})$

Additional file 5: S5. Original blots in fig. 1. (PPTX $1641 \mathrm{~kb}$ )

Additional file 6: $\mathrm{S} 6$ Table Echocardiographic measurements of LV function in the 8 weeks old DTg mice (ASD and non-ASD). (DOCX $14 \mathrm{~kb}$ )

Additional file 7: S7. Original blots in fig. 4. (PPTX $1028 \mathrm{~kb}$ )

\section{Abbreviations}

A: Atria; aMEK1: Active form of human MEK1; ASD: Atrial septal defects; CHD: Congenital heart disease; DOX: Doxycycline; Dpc: Day post copulation; DTg: Double transgenic (DTg); EF: Ejection fraction; ERK1/2: Mitogenactivated protein kinase 1/2; FS: Fractional shortening; HA: Hemagglutinin; i.p.: Intraperitoneal; LV: Left ventricular; MEK1: Mitogen-activated protein kinase kinase 1; MH: aMHC-tTA transgenic mouse; MKP: MAP kinase phosphatase; P-ERK1/2: Phospho-ERK1/2; TAMEK: Transgenic mouse with tetracycline transactivator regulated aMEK1 gene; TetR: Tetracycline repressor protein; Tg: Transgenic mouse; tTA: Tracycline-responsive transcriptional activator; V: Ventricle; a-MHC: a-myosin heavy chain

\section{Acknowledgements}

The authors appreciate the generous gifts of the HA-tagged human active MEK1 CDNA and the transgenic mouse model (MEK1 Tg) provided by Dr. Natalie G. Ahn and Dr. Jeffrey Molkentin.

\section{Funding}

This work was supported by National Institutes of Health; Grant numbers: K08HL079239 and R01HL083118.

\section{Availability of data and materials}

All datasets, on which the conclusions of the manuscript rely on, are presented in the paper.

\section{Authors' contributions}

$\mathrm{CY}$ and MJM conceived the study, $\mathrm{CY}$ was responsible for the design of the research; CY, YF and YY performed the experiments; CY analyzed the data and interpreted the results of the experiments; $C Y$ and YF prepared the figures and the manuscript; CY and MJM revised the manuscript. All authors read and approved the final manuscript.

\section{Ethics approval}

All procedures conformed to the Guide for the Care and Use of Laboratory Animals published by the US National Institutes of Health (NIH Publication No. 85-23, revised 1996), and were approved by the Institutional Animal Care and Use Committee of the University of California, San Francisco.

\section{Consent for publication}

Not applicable.

\section{Competing interests}

The authors declare that they have no competing interests.

Our study doesn't include any data about people or human tissue samples. All animal experiments in this research were reviewed and approved by the

\section{Publisher's Note}

Springer Nature remains neutral with regard to jurisdictional claims in published maps and institutional affiliations.

Received: 21 April 2017 Accepted: 6 November 2017 Published online: 24 November 2017

\section{References}

1. van der Linde D, Konings EE, Slager MA, Witsenburg M, Helbing WA, Takkenberg JJ, et al. Birth prevalence of congenital heart disease worldwide: a systematic review and meta-analysis. J Am Coll Cardiol. 2011;58:2241-7.

2. Schott JJ, Benson DW, Basson CT, Pease W, Silberbach GM, Moak JP, et al. Congenital heart disease caused by mutations in the transcription factor NKX2-5. Science. 1998;281:108-11.

3. Chen Y, Han ZQ, Yan WD, Tang CZ, Xie JY, Chen H, et al. A novel mutation in GATA4 gene associated with dominant inherited familial atrial septal defect. J Thorac Cardiovasc Surg. 2010;140:684-7.

4. Ching YH, Ghosh TK, Cross SJ, Packham EA, Honeyman L, Loughna S, et al. Mutation in myosin heavy chain 6 causes atrial septal defect. Nat Genet. 2005;37:423-8.

5. Mori AD, Bruneau BG. TBX5 mutations and congenital heart disease: Holt-Oram syndrome revealed. Curr Opin Cardiol. 2004;19:211-5.

6. Bueno OF, De Windt LJ, Tymitz KM, Witt SA, Kimball TR, Klevitsky R, et al. The MEK1-ERK1/2 signaling pathway promotes compensated cardiac hypertrophy in transgenic mice. EMBO J. 2000;19:6341-50.

7. Lips DJ, Bueno OF, Wilkins BJ, Purcell NH, Kaiser RA, Lorenz JN, et al. MEK1ERK2 signaling pathway protects myocardium from ischemic injury in vivo. Circulation. 2004;109:1938-41.

8. Yeh CC, Malhotra D, Yang YL, Xu Y, Fan Y, Li H, et al. MEK1-induced physiological hypertrophy inhibits chronic post-myocardial infarction remodeling in mice. J Cell Biochem. 2013;114:47-55.

9. Digilio MC, Lepri F, Baban A, Dentici ML, Versacci P, Capolino R, et al. RASopathies: clinical diagnosis in the first year of life. Mol Syndromol. 2011;1:282-9.

10. Dentici ML, Sarkozy A, Pantaleoni F, Carta C, Lepri F, Ferese R, et al. Spectrum of MEK1 and MEK2 gene mutations in cardio-facio-cutaneous syndrome and genotype-phenotype correlations. Eur J Hum Genet. 2009;17:733-40.

11. Anastasaki C, Rauen KA, Patton EE. Continual low-level MEK inhibition ameliorates cardio-facio-cutaneous phenotypes in zebrafish. Dis Model Mech. 2012;5:546-52.

12. Wang Y, Kim E, Wang X, Novitch BG, Yoshikawa K, Chang LS, et al. ERK inhibition rescues defects in fate specification of Nf1-deficient neural progenitors and brain abnormalities. Cell. 2012;150:816-30.

13. Inoue $\mathrm{S}$, Moriya M, Watanabe $\mathrm{Y}$, Miyagawa-Tomita S, Niihori T, Oba D, et al. New BRAF knockin mice provide a pathogenetic mechanism of developmental 
defects and a therapeutic approach in cardio-facio-cutaneous syndrome. Hum Mol Genet. 2014;23:6553-66.

14. Franco D, Lamers WH, Moorman AF. Patterns of expression in the developing myocardium: towards a morphologically integrated transcriptional model. Cardiovasc Res. 1998;38:25-53.

15. Rothman KJ. No adjustments are needed for multiple comparisons. Epidemiology. 1990;1:43-6.

16. Savitz DA, Olshan AF. Multiple comparisons and related issues in the interpretation of epidemiologic data. Am J Epidemiol. 1995;142:904-8.

17. Perneger TV. What's wrong with Bonferroni adjustments. Br Med J. 1998;316: 1236-8.

18. Bacchetti P. Peer review of statistics in medical research: the other problem. Br Med J. 2002;324:1271-3.

19. Fan Y, Yang Y-L, Yeh C-C, Mann MJ. Spacial and temporal patterns of gene expression after cardiac MEK1 gene transfer improve post-infarction remodeling without inducing global hypertrophy. J Cell Biochem. 2017;118: 775-84.

20. Tanoue T, Adachi M, Moriguchi T, Nishida E. A conserved docking motif in MAP kinases common to substrates, activators and regulators. Nat Cell Biol. 2000;2:110-6.

21. Zakariyah AF, Rajgara RF, Veinot JP, Skerjanc IS, Burgon PG. Congenital heart defect causing mutation in Nkx2.5 displays in vivo functional deficit. J Mol Cell Cardiol. 2017;105:89-98.

22. Chen J, Qi B, Zhao J, Liu W, Duan R, Zhang MA. Novel mutation of GATA4 (K300T) associated with familial atrial septal defect. Gene. 2016;575:473-7.

23. Kodo K, Yamagishi HGATA. Transcription factors in congenital heart defects: a commentary on a novel GATA6 mutation in patients with tetralogy of Fallot or atrial septal defect. J Hum Genet. 2010;55:637-8.

24. Campbell $\mathrm{M}$. Incidence of cardiac malformations at birth and later, and neonatal mortality. Br Heart J. 1973;35:189-200.

25. Saenz RB, Beebe DK, Triplett LC. Caring for infants with congenital heart disease and their families. Am Fam Physician. 1999;59:1857-68.

26. Chattergoon NN, Louey S, Stork PJ, Giraud GD, Thornburg KL. Unexpected maturation of PI3K and MAPK-ERK signaling in fetal ovine cardiomyocytes. Am J Physiol Heart Circ Physiol. 2014;307:H1216-25.

27. Gurjar MV, Deleon J, Sharma RV, Bhalla RC. Role of reactive oxygen species in IL-1 beta-stimulated sustained ERK activation and MMP-9 induction. Am J Physiol Heart Circ Physiol. 2001;281:H2568-74.

28. Aikawa R, Komuro I, Yamazaki T, Zou Y, Kudoh S, Tanaka M, et al. Oxidative stress activates extracellular signal-regulated kinases through Src and Ras in cultured cardiac myocytes of neonatal rats. J Clin Invest. 1997;100:1813-21.

29. Moosavi F, Hosseini R, Saso L, Firuzi O. Modulation of neurotrophic signaling pathways by polyphenols. Drug Des Devel Ther. 2015;10:23-42.

30. Pearce MJ, McIntyre TM, Prescott SM, Zimmerman GA, Whatley RE. Shear stress activates cytosolic phospholipase A2 (CPLA2) and MAP kinase in human endothelial cells. Biochem Biophys Res Commun. 1996;218:500-4.

31. Winston JB, Schulkey CE, Chen IB, Regmi SD, Efimova M, Erlich JM, et al. Complex trait analysis of ventricular septal defects caused by Nkx2-5 mutation. Circ Cardiovasc Genet. 2012;5:293-300.

32. Le Bouffant R, Wang JH, Futel M, Buisson I, Umbhauer M, Riou JF. Retinoic acid-dependent control of MAP kinase phosphatase-3 is necessary for early kidney development in Xenopus. Biol Cell 2012;104:516-532.

33. Eblaghie MC, Lunn JS, Dickinson RJ, Munsterberg AE, Sanz-Ezquerro JJ, Farrell ER, et al. Negative feedback regulation of FGF signalling levels by Pyst1/MKP3 in chick embryos. Curr Biol. 2003;13:1009-18.

34. Kawakami Y, Rodriguez-Leon J, Koth CM, Buscher D, Itoh T, Raya A, et al. MKP3 mediates the cellular response to FGF8 signalling in the vertebrate limb. Nat Cell Biol. 2003;5:513-9.

35. Li C, Scott DA, Hatch E, Tian X, Mansour SL. Dusp6 (Mkp3) is a negative feedback regulator of FGF-stimulated ERK signaling during mouse development. Development. 2007:134:167-76.

36. Maillet M, Purcell NH, Sargent MA, York AJ, Bueno OF, Molkentin JD. DUSP6 (MKP3) null mice show enhanced ERK1/2 phosphorylation at baseline and increased myocyte proliferation in the heart affecting disease susceptibility. J Biol Chem. 2008;283:31246-55.

37. Pouysségur J, Volmat V, Lenormand P. Fidelity and spatio-temporal control in MAP kinase (ERKs) signalling. Biochem Pharmacol. 2002;64:755-63.

38. Corson LB, Yamanaka Y, Lai KM, Rossant J. Spatial and temporal patterns of ERK signaling during mouse embryogenesis. Development. 2003;130:4527-57.

\section{Submit your next manuscript to BioMed Central and we will help you at every step:}

- We accept pre-submission inquiries

- Our selector tool helps you to find the most relevant journal

- We provide round the clock customer support

- Convenient online submission

- Thorough peer review

- Inclusion in PubMed and all major indexing services

- Maximum visibility for your research

Submit your manuscript at www.biomedcentral.com/submit

) Biomed Central 